\title{
An experimental analysis in horizontal convection with IR thermography
}

by G. Ceglia, S. Discetti*

`Dept. of Aerospace Engineering, University of Naples, P.le Tecchio 80- 80125 Napoli, Italy, stefano.discetti@unina.it

\begin{abstract}
The aim of this work is to investigate by means of infrared thermography the features of the boundary layer developing on the bottom base of a water-filled volume where a differential heating input is provided. The circulation induced by piecewise thermal conditions on a horizontal boundary is commonly referred as horizontal convection. In the present study, a uniform temperature is imposed on one half of the bottom base of a Plexiglass ${ }^{\circledR}$ box filled with degassed water, while on the other half a uniform heat flux input is provided; on the other box boundaries, the condition of adiabatic wall is reproduced. The range of tested $\mathrm{Ra}_{\mathrm{L}}$ numbers (referred to the heat flux input and box depth) is $5.54 \times 10^{9} \div 7.63 \times 10^{10}$. The transition of the boundary layer and the development of the time evolution of the coherent structures motion are observed on the half base where the uniform heat flux input is provided. At the same location, the onset of longitudinal streamwise rolls and, subsequently, the rise of a strong vertical plume are followed for all the tested operating conditions.
\end{abstract}

\section{Introduction}

Differential heating along horizontal boundaries of a fluid-filled volume establishes an overturning circulation, commonly referred to as horizontal convection [1]. This peculiar form of natural convection is a suitable model for many natural phenomena, such as the convective motion of the earth mantle, the dynamics of atmospheric structures and of the oceanic overturning circulation and so forth.

The governing dynamics of the horizontal convection can be characterized by the presence of longitudinal stream-wise rolls, as noticed in a large variety of natural convection flows [2, 3]. A necessary condition for the development of such longitudinal vortices is the presence of a force acting perpendicularly to the direction of main fluid motion, in which case, they are referred to as Görtler vortices [2, 4]. In practical applications the mixing induced by the longitudinal vortices is of interest because of its relevance in industrial processes such as a chemical vapor deposition, cooling of electronic apparatus and nuclear reactors, material processing, crystal growth, solar energy collectors and welding.

In the literature, this flow field is only marginally treated for the case of horizontal convection, while it is more widely discussed in the general scenario of natural convection. An early experiment that investigated the formation of longitudinal rolls in the flow pattern on heated inclined plates was carried out by [5]; results show an increase of the number of the rolls across the plate width with increasing the temperature difference between the heated surface and the fluid, regardless of the plate inclination angle. In the experimental investigations performed by [6] a horizontal water channel is uniformly heated from below; results clearly reveal the presence of a secondary flow (in the form of longitudinal rolls) superimposed to the main one along the channel; the temporal evolution of the flow field consists of an initial set of regularly spaced thermal plumes which ascend from the heated surface; subsequently, the plumes bifurcate and curl back towards the heated surface. This pattern determines a significant heat transfer enhancement. The same conclusion was drawn in [2,3], where the local heat-transfer distribution on a flat inclined plate in water, for the case of natural convection under condition of constant heat flux input, is investigated. The heat transfer increase is determined by a non-linear distortion of the boundary layer because of the presence of longitudinal vortices, which may indirectly lead to a laminar-turbulent transition of the flow. Besides, the vortices perturb the boundary layer, forming crests and depressions in the fluid so that the fluid mixing in the boundary layer is improved.

An experiment was carried out by [7] to investigate the structure of the longitudinal vortex rolls in mixed convective air flow through a horizontal flat duct with a circular heated plate embedded in the duct bottom. The observed rolls in the throughflow have not the same size; in fact, they are not completely spanwisely symmetric. Two types of instability are detected: the inertia instability at high Reynolds numbers and buoyancy driven instability at high buoyancyto-inertia ratio. The experimental study and numerical simulations of natural convective flow above partially heated surface in a rectangular enclosed domain was presented by $[9,10]$. Results showed the presence of longitudinal structures arising over the horizontal heated surface. The mechanism of the formation and development of longitudinal rolls allows the enhancement of the heat transfer that occurs at the vortex center. This behavior promotes the laminar/turbulent transition of the flow.

Infrared Thermography can provide a significant contribution in the understanding of the onset of the longitudinal rolls for the case of an instantaneous change of the heating boundary conditions, which determine the development of the horizontal convection. In the present study the experimental setup and the boundary conditions are very similar to that of [11], the main difference being that the heat flux input is provided by a printed circuit board. The relatively low Biot number enables the possibility to measure the board temperature distribution on the opposite surface 
of that of heat exchange by means of an infrared camera. The adopted acquisition system, with a frame rate up to $50 \mathrm{~Hz}$, fully resolves in time the onset and development of the longitudinal rolls. In this work, the temporal evolution of the flow field close to the heated surface is investigated as a function of the imposed Rayleigh number and of the convection cell height; in all cases the condition of free-surface is imposed at the upper boundary.

\section{Experimental setup}

The onset of the longitudinal helical rolls with mixed coherent structures, driven by differential heating along one horizontal boundary, is investigated in a box $\left(L=300 \mathrm{~mm}\right.$ long, $D=150 \mathrm{~mm}$ wide and $H_{B}=150 \mathrm{~mm}$ deep, internal dimensions) whose sketch and reference frame are reported in Fig.1. The box is filled with de-gassed de-mineralized water.

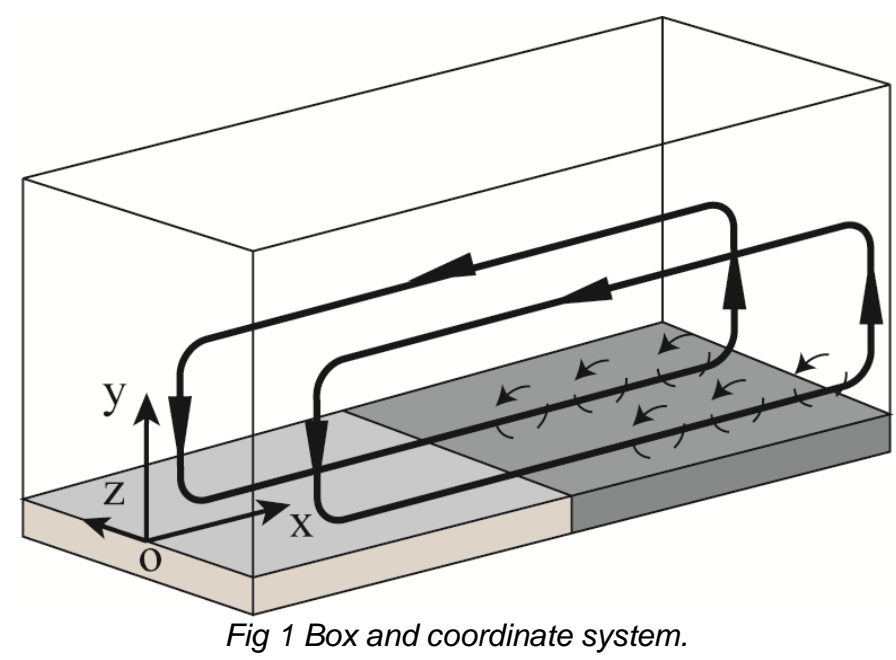

Plexiglass ${ }^{\circledR}$ GS 233 is chosen for the mainframe, due to its suitable surface quality and index of refraction very close to that of water. The desired condition of thermally insulated lateral walls is obtained with a wall thickness of $15 \mathrm{~mm}$, a compromise value to minimize both the effects of thermal losses due to conduction towards the external ambient and along the tangential direction. Plexiglass ${ }^{\circledR}$ is very suitable for this application thanks also to its relatively low electrical conductivity and satisfactory mechanical properties.

The base of the tank is divided in two equal halves, into which a heat exchanger and a printed circuit board are placed. The heat exchanger is constituted by a copper serpentine, silver-soldered to a $10 \mathrm{~mm}$ thick electrolytic copper plate $\left(150 \mathrm{~mm}\right.$ long, $150 \mathrm{~mm}$ wide); the high thermal conductivity of the plate (approximately $390 \mathrm{Wm}^{-1} \mathrm{~K}^{1}$ ) guarantees with very close approximation the desired boundary condition of uniform temperature. Water, at the same initial temperature of the testing fluid and controlled by means of an ultra-thermostat, is pumped through the serpentine.

The printed circuit board determines the desired boundary condition of practically uniform heat flux input on the other half of the box base by means of Joule effect. The outer side of the heating printed circuit board is coated with a thin layer of high emissivity paint $(\varepsilon=0.95)$ in order to improve the signal to noise ratio of the IR camera system. The copper pathways (tracks) of the printed circuit board are $5 \mu \mathrm{m}$ thick, $1.8 \mathrm{~mm}$ wide and placed at a $2 \mathrm{~mm}$ pitch. The total board thickness, including the fiberglass layer, is $0.5 \mathrm{~mm}$. The circuit in-plane dimensions are $150 \times 150 \mathrm{~mm}^{2}$ and its nominal electrical resistance is about $30 \Omega$. A $1 \mathrm{~mm}$ thick support rod reduces the effect of deflection of the printed circuit board due to the hydrostatic pressure.

Thanks to the relatively low thermal resistance of the printed circuit board with respect to that of the convective heat transfer towards water, a very small Bi number is produced, so that it is possible to assume that the temperature is constant across the board thickness, thus enabling the IR measurement by observing the board outer surface.

In the work by [11], an exhaustive theoretical analysis of the governing equations and of the leading dimensionless numbers of the phenomenon is provided. Horizontal convection is driven by three dimensionless parameters: the Prandtl number $\operatorname{Pr}=v / \alpha$, where $v$ and $\alpha$ are the kinematic viscosity and the thermal diffusivity of the fluid, respectively; the aspect ratio $A=H / L$, where $H$ and $L$ are the depth and length of the box, respectively; the Rayleigh number, which can be expressed either in terms of the imposed heat flux or of a temperature difference, according to the nature of the actual boundary conditions.

In the present work, $R a_{L}$ is based on the imposed heat flux:

$R a_{L}=\frac{g \beta F_{T} L^{4}}{\rho c_{p} \alpha^{2} v}$

where $g$ is the gravity acceleration and $F_{T}$ the input heat flux; with regard to the fluid properties, $\beta$ is the thermal expansion coefficient, $\rho$ the mass density and $c_{p}$ the specific heat at constant pressure. 
The power supply is regulated in order to obtain three values of the Rayleigh number $R a_{L}$, namely $5.54 \times 10^{9}$, $2.96 \times 10^{10}$ and $7.63 \times 10^{10}$, and the depth of the volume is varied in order to obtain three values of the aspect ratio $A$, i.e. $0.5,0.25$ and 0.05 .

\subsection{Heat transfer measurement}

The relatively high frequency response of the printed circuit board suffices to follow the temporal evolution of the local convective heat transfer coefficient $h_{f}$, measured by using the heated thin foil sensor [12], i.e. by using the relation:

$h_{f}=\frac{q_{j}-q_{r}}{T_{w}-T_{a w}}$

where $q_{j}$ is the Joule heat flux input, $q_{r}$ the radiative heat flux to ambient, $T_{w}$ is the wall temperature and $T_{a w}$ is the adiabatic wall temperature that coincides with the initial average temperature of the working fluid in the box. The other contributions to the energy balance, i.e. mainly the heat losses at the rear surface of the printed circuit board due to natural convection and tangential conduction contributions, are estimated to be negligible.

Assuming the ambient as a black body at a constant temperature $T_{a}$ (i.e. the average ambient temperature) and that the sensor surface is gray in the wavelength of interest, the net rate of radiation heat loss is estimated with the Stefan-Boltzmann's law:

$q_{r}=\sigma \varepsilon\left(T_{w}^{4}-T_{a}^{4}\right)$

where $\sigma$ is the Stefan-Boltzmann's constant and $\varepsilon$ is the total hemispherical emissivity coefficient of the printed circuit board outer surface.

The test procedure is divided in two steps. First, the $T_{a w}$ is measured by acquiring a set of images without providing electric current to the printed circuit board (these thermograms are commonly defined as cold images); subsequently, when the power supply is switched on, the hot images sequence is recorded to measure the evolution of the wall temperature distribution.

Experimental data are reduced in dimensionless form in terms of Nusselt number $N u=h_{f} L / k$, where $k$ is the water thermal conductivity coefficient.

A front surface (FS) mirror and an IR thermography system (IR camera), based on the CEDIP JADE III MW (FPA of $320 \times 240$ pixels, working in the $4-5 \mu \mathrm{m}$ band) with spatial resolution of about $0.59 \mathrm{~mm} /$ pixel, are employed to measure the temperature distribution of the printed circuit board surface (Fig.2); the radiometric camera calibration takes into account the presence of the FS mirror.

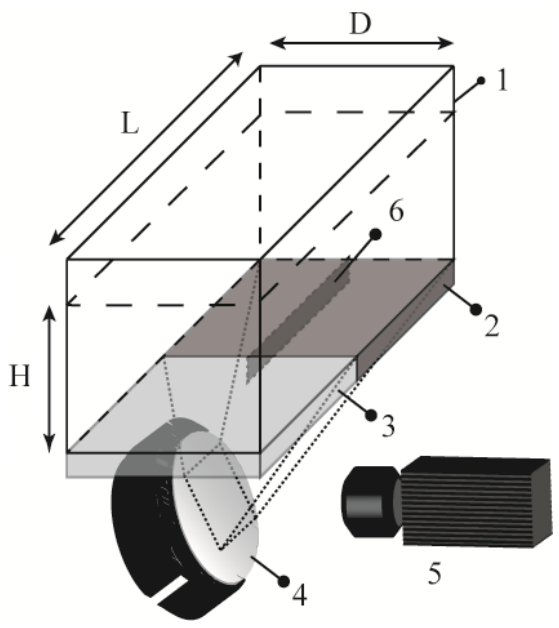

Fig 1 Scheme of the experimental setup: 1 - box, 2 - printed circuit board, 3 - heat exchanger, 4 - FS mirror, 5-IR camera, 6 - support rod.

\section{Heat transfer results}

The initial development stages of horizontal convection, in terms of heat transfer at the horizontal boundary where the heat forcing is applied, are investigated. The instantaneous distributions of the Nusselt number are measured as described in Sec. 2.1.

The features of the initial unsteady regime of horizontal convection adjust, towards the thermal equilibrium state, is strongly dependent on the boundary conditions [1]. As already stated in Sec. 2, the Rayleigh number RaL, the Prandtl number $\operatorname{Pr}$ and the aspect ratio $A$ are the main parameters characterizing the onset of the horizontal convection; besides, 
it should be underlined that, for the designed experimental apparatus, the mean temperature of the water is strongly affected by the imposed heat flux, leading to a variation of the Prandtl number $\operatorname{Pr}$ in the analyzed range of Rayleigh number $R a_{L}$. For this reason, a preliminary calibration of the apparatus has been performed by using a thermometer to estimate the mean water temperature in all the tested conditions. Considering that reference [11] shows the temperature profile quite flat and pretty independent of the $x$-position (longitudinal abscissa), a good approximation of the mean temperature can be obtained with a limited number of measurements far enough from the base.

When the power supply is switched on, the density stratification of the water over the printed circuit board surface determines the establishment of a vertical convective circulation, in the form of local ascending plumes as in the classical Rayleigh-Bénard convection cells (R-B cells) scenario. The distribution of the cells is distinctly visible in Figs. 35 , which show, for $A=0.5$, snapshots of the Nusselt number distribution for the three different tested Rayleigh numbers at given instants after the power supply activation. As the Rayleigh number $R a_{L}$ increases, the R-B cells become smaller (i.e. their mean diameter decreases) and the picks of the local $N u$ number increase both in intensity as well as in their number.

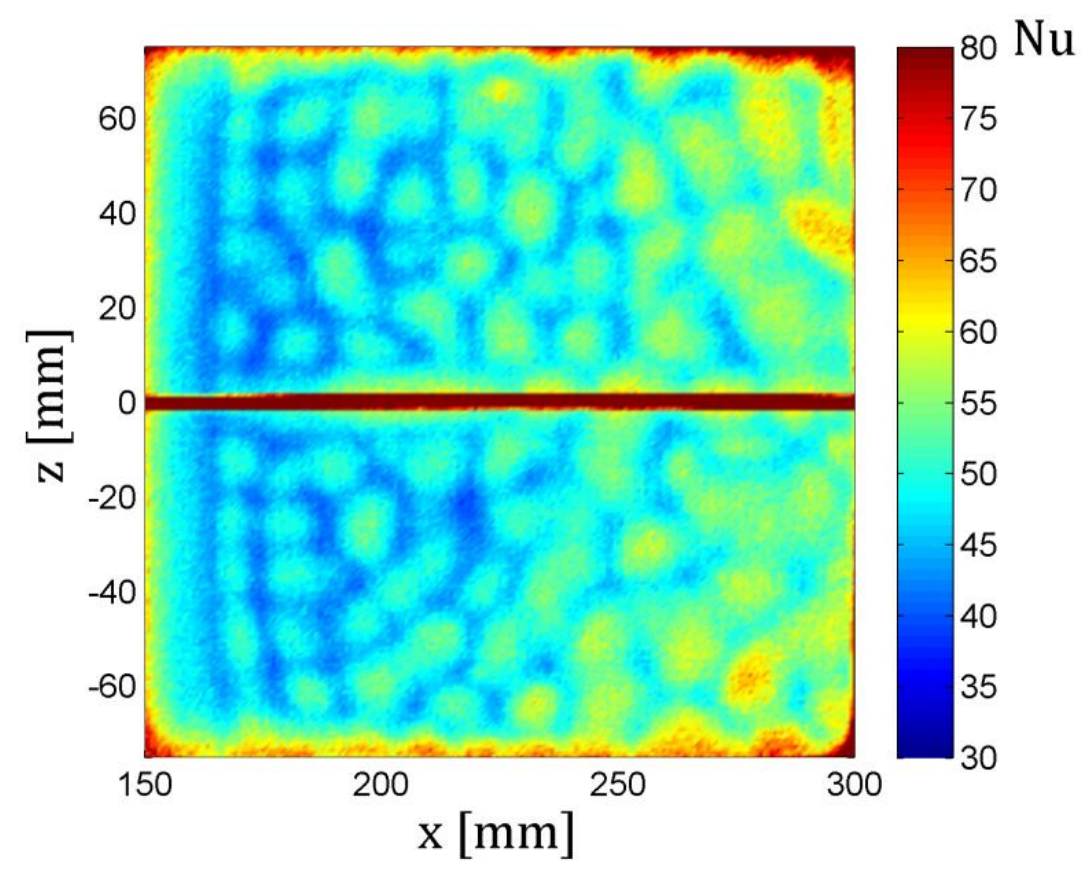

Fig. 3 Nu number map on the printed cirtuit board surface for $R a_{L}=5.54 \times 10^{9}$ and $A=0.5$, measured after $1 \mathrm{~min}$, 22s. 


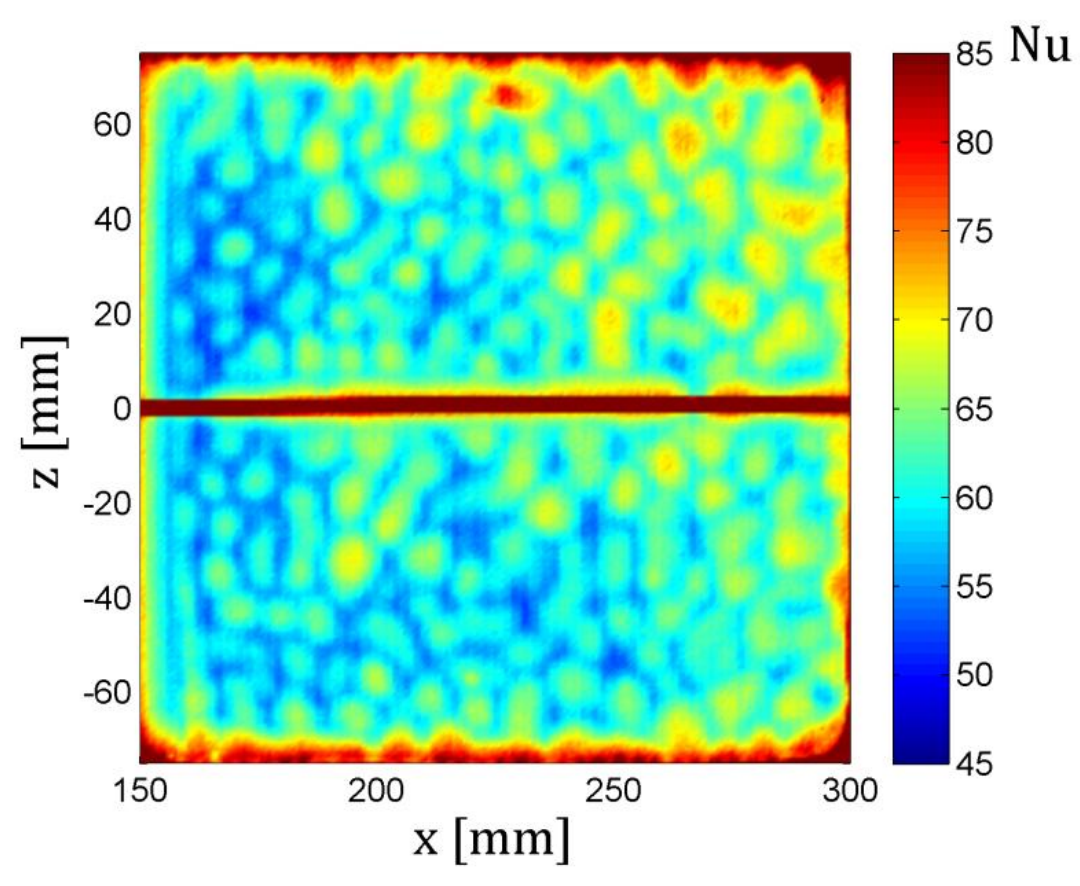

Fig.4 Nu number map on the printed cirtuit board surface for $R a_{L}=2.96 \times 10^{10}$ and $A=0.5$, measured after 39 s.

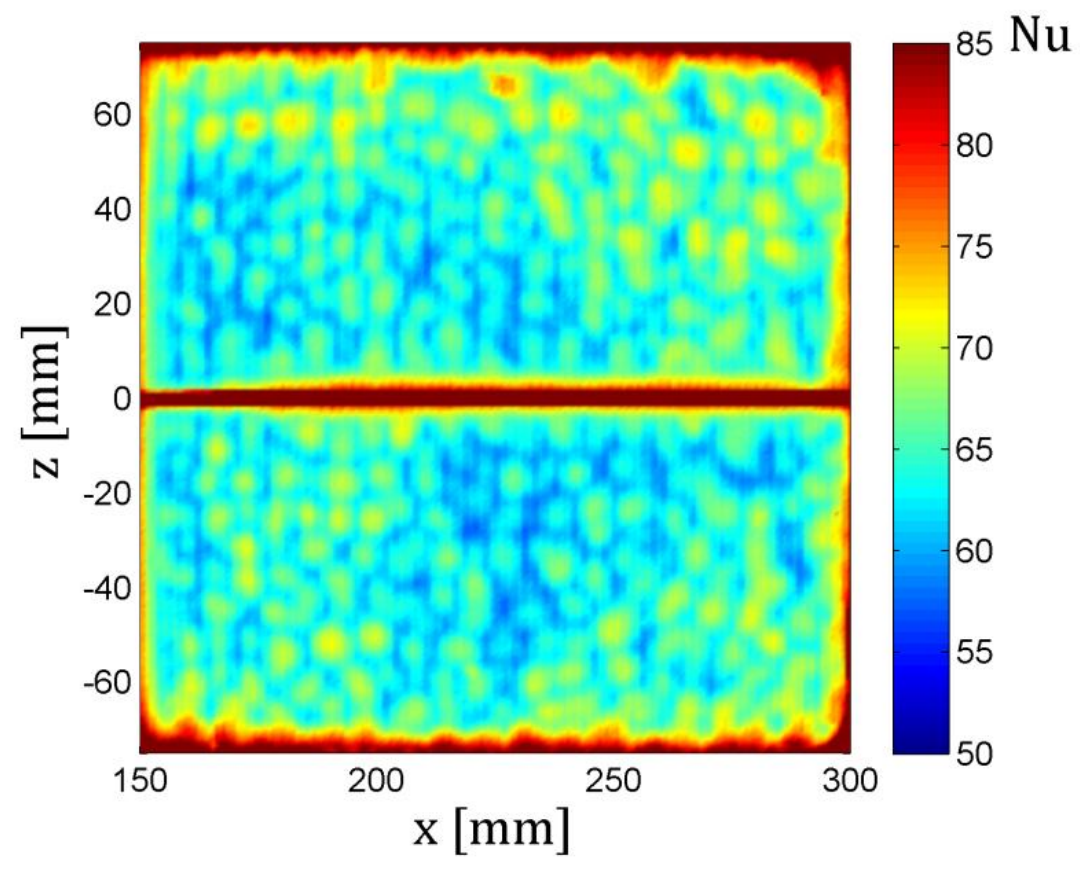

Fig. $5 \mathrm{Nu}$ number map on the printed cirtuit board surface for $R a_{L}=7.63 \times 10^{10}$ and $A=0.5$, measured after $26 \mathrm{~s}$.

The different stages of the onset of longitudinal convection, which later occurs, are reported in Fig. 6. Initially, the field is dominated by the presence of R-B cells (Fig.6a); in the meanwhile cold water entrained by the regions immediately above the heat exchanger contributes to the flow of the nearby cells in providing fluid to compensate the vertical mass flow rate due to the ascending plumes. This effect determines the development of the weak horizontal motion from left to right (see left side of Fig. 6a), resulting in the development of a boundary layer on the printed circuit board

Then, the vertical penetration of the plumes progressively increases in intensity requiring more fluid to be supplied; this effect inflates the intensity of the horizontal motion. In the meantime, longitudinal instabilities determine the onset of longitudinal rolls, which sweep away the R-B cells upon the printed circuit board surface. At this stage of 
development, the Nu number distributions clearly highlight the transition of the boundary layer to turbulent regime (see the abrupt Nu number increase at the right side of Fig. 6b-c).

The longitudinal rolls influence the heat transfer significantly ([5], [9]), thus the pattern of local Nu number on the printed circuit board represents a footprint of the rolls themselves. As a matter of fact, in the down-wash region of each rolls pair, the heat transfer is enhanced while, in the up-wash region, is decreased (the core of each longitudinal roll can be easily identified on the map as a strip of local minimum heat transfer).

In Fig. 6c, when the steady state regime is practically established, the presence of the wall and the intense density stratification force the fluid to separate from the bottom surface and to arrange itself in a complex pattern of turbulent ascending plumes; this determines a decrease of the longitudinal extension of the rolls, as it is evident in the $\mathrm{Nu}$ number map.
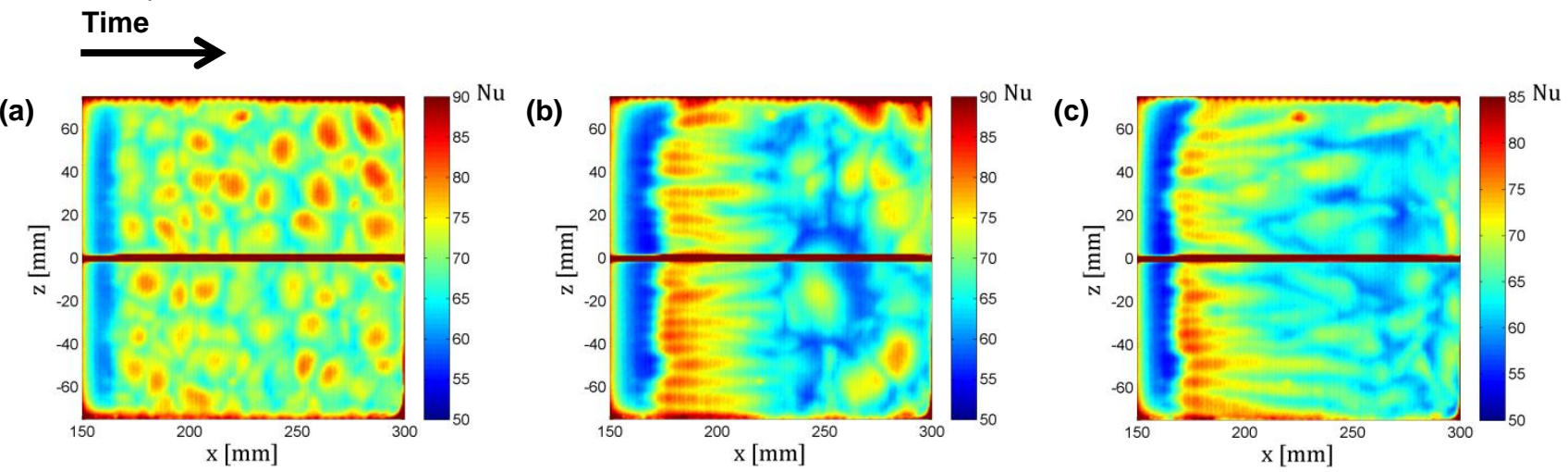

Fig. 6 Measured Nu number maps upon the printed circuit board for $R a_{L}=7.63 \times 10^{10}$ and $A=0.5$ in the transient stage: (a) onset of instability flow region and Rayleigh-Bénard cells coalescence (37 s), (b) development of longitudinal rolls, sweeping away the Rayleigh-Bénard cells (1 min), (c) fully developed longitudinal rolls stage (4 min, $37 \mathrm{sec}$ ).

In Fig. 7, the measured Nu maps over the printed circuit board surface are shown for three different Rayleigh number $R a_{L}$ at the previously described last stage of the transient development of the horizontal convection, i.e. when the longitudinal rolls system is, in a sense, fully developed. For each operating value of the power supply, it is possible to distinguish three different flow field regions: the laminar boundary layer development, the longitudinal rolls and the separated flow due to the presence of the ascending plumes.

Buoyancy forces in the laminar region are not intense enough to overcome the stabilizing effects of the fluid viscosity, thereby the heat transfer distribution appears quite uniform along the spanwise direction.

The laminar boundary layer region ends when the buoyancy forces, acting perpendicularly to the main flow, impose the onset of longitudinal instability. The boundary layer perturbation result in the appearance of longitudinal rolls at the transition point. As the $R a_{L}$ number increases (and, accordingly, the buoyancy forces become more and more intense), the transition longitudinal abscissa moves upstream and, furthermore, the number of longitudinal rolls increases along the spanwise direction. The buoyancy forces supply energy to the longitudinal rolls, which increase the water circulation between the heated surface and the interior of the box, resulting in a more effective heat transfer on the printed circuit board surface.

As the longitudinal rolls approach the sidewall (right side of the Nusselt number maps), the buoyancy forces determine the Breakdown of the longitudinal rolls into a complex turbulent vertical plumes system. As the Rayleigh number $R a_{L}$ increases, the longitudinal rolls are stretched and distorted and, furthermore, the heat transfer decreases because of the separation along the right sidewall.
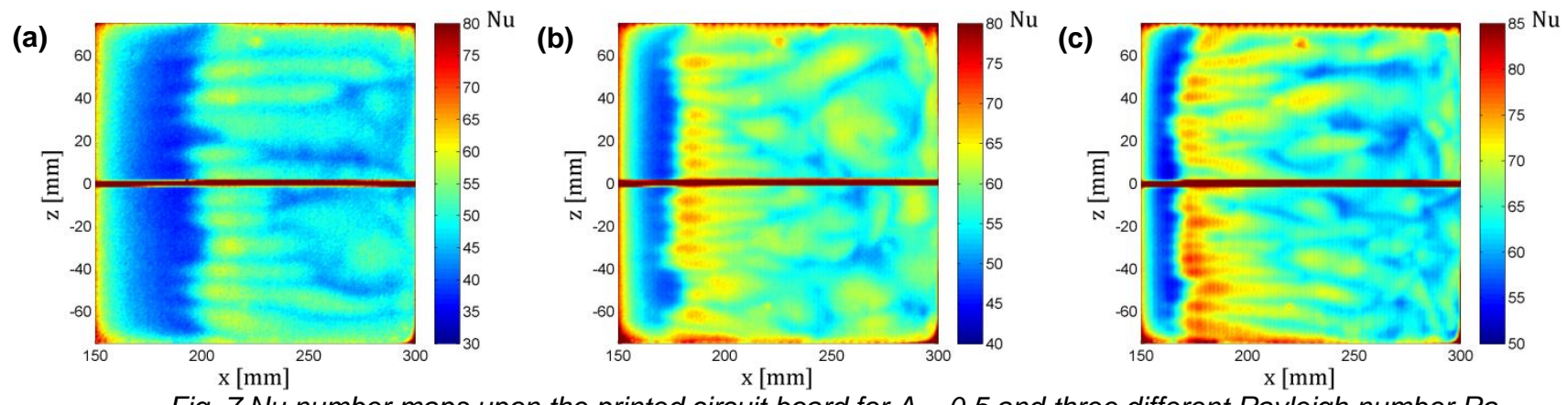

Fig. 7 Nu number maps upon the printed circuit board for $A=0.5$ and three different Rayleigh number Ra number values: (a) $R a_{L}=5.54 \times 10^{9}$ measured after $4 \mathrm{~min}, 51 \mathrm{~s}$, (b) $2.96 \times 10^{10}, 4 \mathrm{~min}, 57 \mathrm{~s}$, (c) $7.63 \times 10^{10}, 4 \mathrm{~min}, 34 \mathrm{~s}$.

Fig. 8 shows the effect of the aspect ratio $A$ for the values $0.05,0.25$ and 0.5 , and for $R a_{L}=2.96 \times 10^{10}$, when the horizontal convection is practically fully developed. 
In Fig. 8a, the measured Nu number map clearly highlights that in the case of low aspect ratio the heat transfer enhancement due to the presence of the longitudinal rolls is less relevant. In this case the longitudinal rolls are characterized by an ordered structure extended along almost all the longitudinal length of the printed circuit board. As the aspect ratio $A$ increases, the larger vertical penetration of the vertical plumes requires a higher mass flow rate; for this reason, the curvature imposed to the horizontal mean flow is more intense, determining the increase of the longitudinal instability. Accordingly, the laminar boundary layer region decreases its extension, i.e. the transition longitudinal abscissa moves upstream. The intensity of the longitudinal rolls and the average heat transfer increase according with results reported in [9]; in particular the motion is characterized by an increased mixing of the fluid and a less ordered condition of the structure, i.e. the longitudinal rolls tend to oscillate along the spanwise direction. .However, the oscillating behavior can be due to the presence of small experimental disturbances, which can alter the inherently steady state of the flow, as already observed by [6].
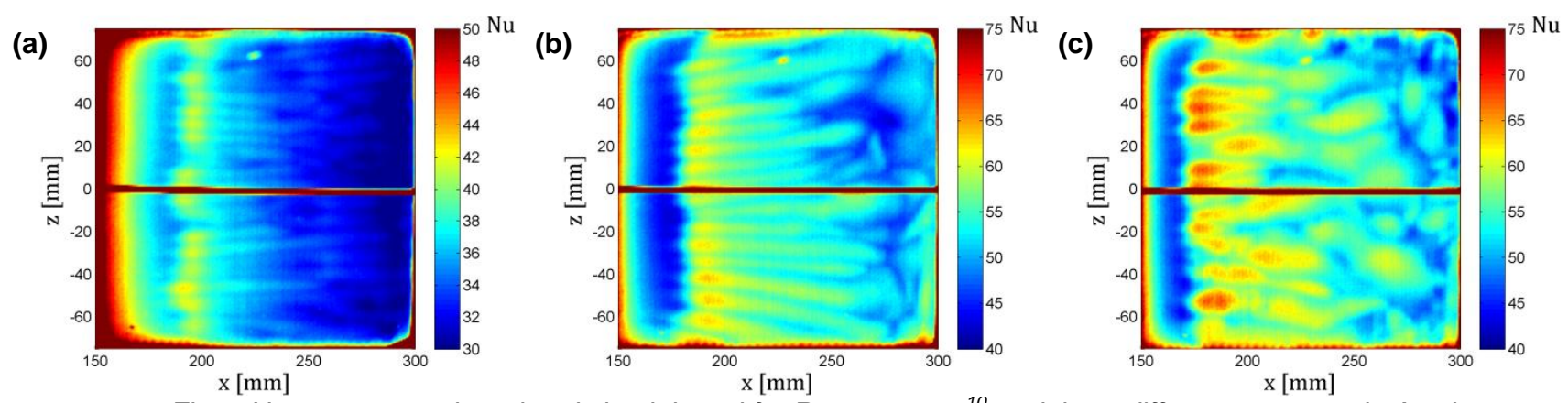

Fig. $8 \mathrm{Nu}$ maps upon the printed circuit board for $R a_{L}=2.96 \times 10^{10}$ and three different aspect ratio $A$ values:

(a) $A=0.05$ measured after $9 \mathrm{~min}, 35 \mathrm{~s}$, (b) 0.25, 9min, 31s, (c) 0.5, 9min, $10 \mathrm{~s}$.

\section{Conclusions}

The establishment of the horizontal convection regime (i.e. natural convection driven by differential thermal boundary condition along the horizontal boundary), due to an abrupt variation of heat flux input, is investigated by means of infrared thermography. The heated thin foil sensor enables the time-resolved measurement of the heat transfer between the flowing fluid and the surface on which the heat input is provided.

Results reveal three subsequent stages: the presence of Rayleigh-Bénard cells over the half heated base of the box, coupled with a weak horizontal motion contributing to the flow rate supply to the vertical convective plumes nearby the cold plate; the onset of longitudinal instabilities and the development of longitudinal rolls in the boundary layer, with increasing streamwise extension over the printed circuit board surface; the final stage in which the R-B cells are completely swept away, and the longitudinal rolls extend towards the vertical sidewall until they separate and merge together in a complex system of turbulent ascending plumes. Results clearly confirm the leading role that the longitudinal rolls play in the enhancing of mixing and heat transfer close to the bottom horizontal boundary. The higher is the heat input, the higher is the average value of the $N u$ peaks over the heat input surface and the larger is the number of longitudinal rolls.

The role of the aspect ratio between height and width of the flow-filled volume is also discussed. Results point out that higher aspect ratios determine larger longitudinal instabilities, reducing the extension of the laminar boundary layer region. The number of longitudinal rolls, however, appear to be aspect ratio invariant in the investigated range.

\section{REFERENCES}

Hughes G.O. and Griffiths R.W. "Horizontal convection”. Annu. Rev. Fluid Mech., vol. 40, pp. 185-208, 2008.

Jeschke P., Biertümpfel R. and Beer H. "Liquid-crystal thermography for heat-transfer measurement in the presence of longitudinal vortices in a natural convection flow". Meas. Sci. Technol., vol. 11, pp. 447-453, 2000.

Jeschke P., Biertümpfel R. and Beer H. "Natural convection heat transfer increase at the laminar-turbulent transition in the presence of instationary longitudinal vortices". International Journal of Heat and Mass Transfer, vol. 46, pp. 3109-3117, 2003.

Saric S. "Görtler Vortices". Annu. Rev. Fluid Mech., vol. 26, pp. 379-409, 1994.

Sparrow E.M. and Husar R.B. "Longitudinal vortices in natural convection flow on inclined plates". J. Fluid Mech., vol. 37, part 2, pp.251-255, 1969.

Maughan J.R. and Incropera F.P. "Secondary flow in horizontal channels heated from below". Experiments in Fluids, vol. 5, pp.334-343, 1987.

Tuh J.L. and Lin T.F. "Structure of mixed convective longitudinal vortex air flow driven by a heated circular plate embedded in the bottom of a horizontal flat duct". Int. J. Heat Mass Transfer, vol. 46, pp. 1341-1357, 2003.

Chin-Tai C. "Thermal instability in natural convection flow over a boundary layer subject to external electrical and magnetic fields". Heat Mass Transfer, vol. 45, pp. 1589-1596, 2009. 
[9] Batalov V.G., Sukhanovskii A.N. "Experimental Investigation of Helicoidal Rolls in an Advective Flow over a Hot Horizontal Surface”. Fluid Dyn., vol. 42, pp.540-549, 2007.

[10] Sukhanovskii A.N., Batalov V.G., Teymurazov A. and Frick P. "Horizontal rolls in convective flow above a partially heated surface”. Eur. Phys. J. B, vol. 9, pp. 85-97, 2012.

[11] Mullarney J.C., Griffiths R.W., Hughes G.O. "Convection driven by differential heating at a horizontal boundary". J. Fluid Mech., vol. 516, pp. 181-209, 2004.

[12] Astarita T., Cardone G., Carlomagno M.G. "Infrared thermography: an optical method in heat transfer and fluid flow visualization”. Optics Lasers Eng., vol. 44, pp. 261-281, 2006. 